\title{
Mise en œuvre de l'Éducation à la Santé Familiale (ESF) en Lorraine
}

\author{
Andréanne de Montfalcon ${ }^{1}$, Jean-François d'Ivernois ${ }^{1}$, Sébastien Riquet ${ }^{1,4}$, François Frété ${ }^{2}$, \\ Jean-Louis Deutscher ${ }^{3}$, Rémi Gagnayre ${ }^{1}$ et Aude-Marie Foucaut ${ }^{1{ }^{*}}$ \\ ${ }^{1}$ Laboratoire Éducations et Pratiques de Santé (EA3412), UFR SMBH, Université Paris 13 - Sorbonne Paris Cité, Bobigny, France \\ ${ }^{2}$ Caisse Centrale de la Mutualité Sociale Agricole, Bobigny, France \\ ${ }^{3}$ Mutualité sociale agricole (MSA) Lorraine, Vandœuvre-lès-Nancy, France \\ ${ }^{4}$ Aix-Marseille Université, École universitaire de maïeutique Marseille Méditerranée, Marseille, France
}

Reçu le 1 décembre 2017 / Accepté le 11 avril 2018

\begin{abstract}
Résumé - Introduction: Les limites des actions de prévention primaire, les déserts médicaux, la saturation et l'inadéquation du recours aux soins d'urgence sont autant de constats amenant à proposer un nouveau concept innovant: l'Éducation à la Santé Familiale (ESF). Cette nouvelle Éducation en santé se propose de délivrer aux familles des compétences de gestion des problèmes de santé courants et de situations d'urgences. Objectif : Cet article présente l'évaluation de la mise en œuvre de l'ESF en Lorraine du point de vue des intervenants et des participants. Méthodes: Après une formation, les intervenants ont animé les modules d'ESF auprès des participants volontaires de trois territoires. La perception de leur formation à l'ESF, leur perception du concept d'ESF et leur point de vue sur l'organisation des ateliers sont évalués. La satisfaction, le sentiment de compétence et d'influence face à des situations problèmes des participants à la formation ESF sont également renseignés. Résultats : Les intervenants sont prêts à réitérer l'expérience. Les participants, très satisfaits, se sentent compétents et ont le sentiment qu'ils auront de l'influence sur les diverses situations complexes qu'ils pourraient rencontrer. Discussion : Le déploiement de l'ESF dans d'autres régions fera l'objet d'une analyse à partir des adaptations pédagogiques et organisationnelles qui découlent de cette expérimentation. Conclusion : Cette étude contribue à montrer l'intérêt de l'intégration de l'ESF dans le champ des éducations en santé afin de répondre aux enjeux sanitaires et sociaux actuels.
\end{abstract}

Mots clés : éducation à la santé familiale / compétences en santé / pédagogie / étude pilote

\begin{abstract}
Implementation of Family Health Education in Lorraine. Introduction: Due to limited primary prevention actions, to the lack of health care provision and the saturation and the misuse of emergency services, it becomes necessary to offer a new innovating concept in health education. The Family Health Education (ESF) helps individuals to learn how to handle by themselves usual health problems and emergency situations. Objective: This article deals with the implementation of such an educational program in Lorraine, from the trainers' and the participants' point of view. Methods: After a training session, the trainers organized classes for voluntary participants in three different cities. Their opinion about the quality and organization of classes was assessed and also their point of view about this new educational concept itself. Results: Trainers are ready to start again this experience. The participants are satisfied and feel themselves competent enough to handle complex situations they might encounter in the future. Discussion: Thanks to this experiment, Family Health Education will be developed in other areas after some adjustments in terms of teaching methods and organization. Conclusion: This study contributes in showing how important this type of educational program is, in order to answer the health and social issues that are currently at stake.
\end{abstract}

Keywords: family health education / health competencies / pedagogy / pilot study

\footnotetext{
*Auteur de correspondance :

audemarie.foucaut@univ-paris13.fr
} 


\section{Introduction}

Le contexte socio-sanitaire rend nécessaire la réappropriation par la famille de la santé de ses membres et la maîtrise des compétences correspondantes, compte tenu des limites actuelles du système de soins en termes d'accessibilité et de prise en charge.

En effet, les politiques de santé actuelles tendent à médicaliser la prévention au lieu d'agir sur le rôle déterminant des comportements individuels en santé [1,2]. Pourtant, ces déterminants sociaux, économiques et culturels semblent avoir davantage d'influence sur l'état de santé des populations qu'en ont les soins médicaux [1]. De même, plusieurs auteurs ont proposé que les secteurs sanitaire et éducatif s'allient [3] en articulant la prévention et le soin [4]. Ainsi, l'individu et son entourage, la famille, devraient pouvoir être reconnus comme de réels producteurs et acteurs de la santé.

Le modèle traditionnel de l'éducation en santé, encore utilisé par de nombreux professionnels de santé, montre ici ses limites car il n'apporte pas les résultats souhaités en santé individuelle ou collective [5]. Par exemple, la proportion de Français décédés à la suite d'un accident de la vie courante devrait pouvoir être réduite [6] grâce à une éducation appropriée. En effet, ces accidents constituent la troisième cause de mortalité en France, après les cancers et les maladies cardio-vasculaires [6], et une des principales problématiques domestiques chez les jeunes de moins de 15 ans [7]. Mais d'un autre côté, seulement $40 \%$ des Français sont formés aux gestes de premier secours [8] et $80 \%$ des personnes de plus de 60 ans doivent être aidés au quotidien par un proche à domicile [9].

D'autre part, le constat est fait d'une réelle désertification de certains territoires par les médecins traitants [7,10,11]. Les soins de premiers recours pâtissent d'un non-renouvellement des médecins généralistes en retraite et d'un manque de nouveaux médecins souhaitant s'installer dans les territoires ruraux, augmentant ainsi les inégalités sociales de santé $[12,13]$. Or, le «pacte territoire santé 2015-2017» souhaite garantir à tous les citoyens un accès aux soins d'urgences et de premier recours en moins de 30 minutes [14].

Parallèlement, on observe une surcharge des consultations de médecine générale pour des troubles mineurs (augmentant le délai d'accès pour d'autres patients) ainsi qu'un engorgement et une saturation des services d'urgences. Cette «sur utilisation », notamment par les parents d'enfants de moins d'un an et les personnes âgées de plus de 75 ans, se caractérise par une inadéquation fréquente du recours au soin d'urgence par rapport à la réelle gravité de la situation $[13,15]$. Une grande partie des consultations non programmées chez le généraliste et des «fausses » urgences, ainsi que les surcoûts engendrés, pourraient être évités si la population pouvait bénéficier d'un apport de connaissances et de compétences sur la prise en charge des problèmes de santé courants, des soins de puériculture et de la petite traumatologie à domicile [16,17].

Ces différents constats appellent un renouveau de l'éducation en santé pour faire face aux enjeux sanitaires et sociaux sous-jacents. Dans cette perspective, le modèle de l'Éducation à la Santé Familiale (ESF) [18,19] serait susceptible d'apporter une solution. En effet, selon d'Ivernois et Gagnayre [20], «le moyen par lequel, nous semble-t-il, on peut rendre une population consciente, connaissante, compétente et responsable vis-à-vis de sa santé est l'éducation ». Par l'apport de connaissances et de compétences techniques aux familles, afin de «prendre soin » et de «porter secours », l'individu et son entourage (la famille) devenant acteurs de leur santé pourraient faire face aux différents problèmes que nous avons évoqués. Ce nouveau concept dans le champ des éducations en santé a été défini par le Laboratoire Éducations et Pratiques de Santé (LEPS EA3412) de l'Université Paris 13 [18,20]. L'ESF concerne les domaines de la prévention, des soins et du secourisme élargi à l'espace familial et de proximité de vie. Ce dispositif a «un caractère opérationnel et d'application immédiate, formant à une vigilance applicable au quotidien comme aux situations d'exception. Il prépare à repérer et faire face aux situations d'urgence mais aussi aux problèmes de santé courants et aux petits maux de la vie quotidienne» [18]. Plus concrètement, l'ESF est une formation, réalisée par des professionnels de soins primaires (et du social au besoin) qui s'adresse aux familles volontaires. Elle forme à une vigilance applicable au quotidien, comme aux situations d'exception (canicule, grand froid, épidémies, etc.); elle les prépare à repérer et faire face aux situations d'urgence, mais aussi aux problèmes de santé courants et aux petits maux de la vie quotidienne [18]. Ce dispositif concourt ainsi à redonner aux familles des compétences de gestion des problèmes de santé courants et des situations d'urgences familiales, permettant notamment de limiter le recours systématique aux urgences hospitalières.

Grâce à l'impulsion du LEPS, de l'Union nationale des familles (UNAF), de la Mutualité sociale agricole (MSA) et des Maisons familiales et rurales (MFR), ce modèle d'éducation en santé se déploie sur plusieurs territoires français [21]. Depuis 2015, l'ESF est expérimentée dans trois territoires de la région Lorraine.

\section{Objectifs}

Le but de cette étude a été d'évaluer la mise en œuvre de la phase de déploiement de l'ESF en Lorraine. Pour cela, nous avons recueilli d'une part, l'opinion des intervenants éducateurs vis-à-vis de la formation méthodologique qui les a préparés à la conduite de l'ESF, leur perception du concept d'ESF et leur point de vue sur la mise en place et le déroulement des formations ESF auprès des familles de Lorraine. D'autre part, nous avons évalué la perception des personnes participant à la formation ESF et leur sentiment de compétence suite à cette formation qu'ils ont reçue. Nous inférons que des satisfactions et des opinions favorables à différents niveaux de la mise en œuvre de l'ESF permettent d'envisager la possibilité d'étendre l'ESF à d'autres régions.

\section{Méthode}

\subsection{Dispositif de formation à l'Éducation à la Santé Familiale}

L'ESF est une formation modulaire [21]. Elle est composée d'un tronc commun obligatoire de 10 à $12 \mathrm{~h}$ dont les thèmes abordés sont «santé et famille au quotidien», «traumatismes et agressions extérieurs», «urgences et secourisme» et « situations d'exception ». Des modules spécifiques s'ajoutent 
au tronc commun, pour 3 à $4 \mathrm{~h}$ de formation supplémentaire. Les participants en formation ont le choix entre "puériculture et pédiatrie », «agir en tant que jeune» et «personnes âgées et gérontologie ». Les intervenants sont principalement des médecins généralistes et urgentistes, des infirmiers, pharmaciens et pompiers, secouristes pour le tronc commun et des pédiatres, sages-femmes, puéricultrices, éducateurs, psychologues, ou encore infirmiers pour les modules spécifiques. La pédagogie active et participative utilisée, notamment par des tables rondes, échanges, cartes questions-réponses, démonstrations et ateliers avec pratiques réajustées, a pour objectif d'accompagner les participants vers l'acquisition ou le renforcement de leurs compétences [22,23].

\subsection{Déroulement de la formation méthodologique des intervenants éducateurs en ESF}

Du 28 au 30 janvier 2015 s'est tenue à Nancy une session de formation méthodologique à l'ESF pour 22 professionnels de santé et du champ social. Ces futurs intervenants éducateurs ont été formés à la philosophie et à la pédagogie de l'ESF en 3 jours pendant lesquels ils ont simulé des situations d'éducation d'ESF en prenant appui sur un panel d'outils pédagogiques (diaporama interactif commun à toutes les formations ESF, cartes questions-réponses, questionnaires d'évaluation). Ces outils destinés à être utilisés et distribués aux familles ont été élaborés par l'IPCEM, le LEPS EA3412, l'Institut national de prévention et d'éducation pour la santé (Inpes) et la Sécurité Civile.

\subsection{Déroulement de la formation ESF auprès des participants}

Les objectifs généraux des modules communs étaient d'être capable de gérer au quotidien les maux de tête, de ventre, les vomissements, la toux, la diarrhée, le rhume, les douleurs, la fièvre, les boutons et les conjonctivites; de savoir constituer une trousse à pharmacie, d'appliquer des mesures de prévention et de prendre en charge des coupures, brûlures, chutes et ampoules. D'autres interventions avaient pour objectif de faire acquérir des compétences de pansage et de bandage, d'alerte des secours, de mise en position latérale de sécurité (PLS) et de massage cardiaque. Les situations d'exception telles que la canicule, le grand froid, les épidémies, les catastrophes ont également été abordées. Des conseils en hygiène et santé ont été donnés. Dans chacune des villes, et avec le soutien de la MSA, l'organisation du recrutement des participants était à la charge des structures ou des collectivités accueillant la formation ESF.

En Meurthe et Moselle, une formation a eu lieu à Lunéville, entre le 24 et le 31 mars 2016, auprès des habitants en insertion socio-professionnelle. Les inscrits ont choisi le module spécifique "puériculture et pédiatrie ».

Dans la Meuse, la formation s'est déroulée sous la supervision du réseau territorial Appui, Dispositif et Outils pour les équipes de premiers Recours (ADOR). Un groupe de jeunes en insertion professionnelle a été formé à Montmédy entre le 28 novembre 2015 et le 9 janvier 2016. Ils ont choisi d'aborder le module spécifique "puériculture et pédiatrie».
Enfin, à Stenay, la formation ESF a été effectuée auprès de deux groupes d'adolescents. Cette formation s'est déroulée au cours d'un trimestre de l'année scolaire 2015-2016 dans un collège, les mercredis après-midi et les fins d'après-midi. Le module spécifique a été : «agir en tant que jeune», permettant d'identifier les ressources à utiliser pour soi et pour sa famille dans des cas aussi divers que le racket, l'anorexie, ou encore la consommation d'alcool.

\section{4 Évaluation de la formation ESF}

\subsubsection{Auprès des intervenants éducateurs}

À la fin de la formation à Nancy, deux questionnaires ont été administrés aux intervenants éducateurs. Le premier questionnaire de 10 items concernait les aspects pédagogiques de la formation à l'ESF; il utilisait une échelle de réponse nuancée : Likert à 4 degrés. Le second questionnaire comprenait 15 items utilisant la même échelle de réponse (Likert à 4 degrés) et 2 questions ouvertes. Celui-ci avait pour objectifs d'évaluer l'opinion des intervenants éducateurs sur l'organisation de la formation (6 items) et sur le choix des formateurs (3 items), leur perception de l'intérêt des différents modules (4 items) et leur appréciation générale sur l'utilité de la formation ( 2 items). De plus, les intervenants étaient interrogés sur les thèmes qui pourraient manquer dans le programme ESF, ainsi que sur leur propre intérêt à participer à ce projet.

La formation ESF auprès des familles a été animée en binôme par les intervenants éducateurs afin d'assurer une double assise - pédagogique et médicale - auprès des participants. Immédiatement après la mise en œuvre de la formation ESF, un questionnaire a été distribué aux intervenants. Il avait pour but d'évaluer par des questions ouvertes, la mise en place des séances d'ESF (2 questions), leur déroulement (2 questions), l'ambiance de la formation (1 question) et leur jugement général sur la formation $\operatorname{ESF}$ (3 questions).

\subsubsection{Auprès des participants}

À la fin de la formation ESF, deux questionnaires ont été distribués aux participants. Le premier évaluait leur perception de la formation ESF. Il était composé de 15 items, avec échelle de réponse nuancée, de type : Likert à 4 degrés.

Le second évaluait le sentiment de compétence que les participants estimaient avoir acquis, à la fin de la formation ESF. Ce sentiment de compétence a été évalué à nouveau par le même questionnaire à distance de la formation : à 3 mois puis à 9 mois. Le questionnaire était composé d'une première partie évaluant le sentiment de compétence des participants (26 items, échelle de Likert à 4 degrés), c'est-à-dire le jugement que les participants portaient sur leurs capacités à exécuter des actions de santé. La seconde partie évaluait le sentiment d'influence face à des situations problèmes par 8 items : pour chacun d'entre eux, le participant notifiait sur une échelle de 0 à 10 - de type EVA - s'il sentait que ce qu'il pouvait faire n'aurait pas (de 0 à 5), ou aurait une influence sur la situation (de 6 à 10). Cette partie du questionnaire correspondait donc au jugement que le participant portait sur sa capacité à influer sur des situations de santé. Le sentiment d'influence peut être assimilé au sentiment 
Tableau 1. Récapitulatif des questionnaires utilisés.

Table 1. Summary of questionnaires used.

\begin{tabular}{|c|c|c|c|c|c|}
\hline & \multicolumn{2}{|c|}{ Questionnaires } & \multicolumn{3}{|c|}{ Temporalité } \\
\hline & But & Format & $\begin{array}{l}\text { Fin de formation } \\
\text { méthodologique des } \\
\text { intervenants en ESF }\end{array}$ & $\begin{array}{l}\text { Fin de formation } \\
\text { ESF auprès des } \\
\text { participants }\end{array}$ & $\begin{array}{l}\text { À distance de la } \\
\text { formation } \\
\text { ESF ( } 3 \text { et } 9 \text { mois) }\end{array}$ \\
\hline \multirow{3}{*}{$\begin{array}{l}\text { Intervenants } \\
\text { éducateurs }\end{array}$} & $\begin{array}{l}\text { Satisfaction de la formation } \\
\text { méthodologique }\end{array}$ & 10 items, Likert à 4 degrés & $\mathrm{x}$ & & \\
\hline & $\begin{array}{l}\text { Opinion sur la formation } \\
\text { méthodologique }\end{array}$ & $\begin{array}{l}15 \text { items, Likert à } 4 \text { degrés; } \\
2 \text { questions ouvertes }\end{array}$ & $\mathrm{x}$ & & \\
\hline & $\begin{array}{l}\text { Évaluation de la mise en } \\
\text { place des séances auprès des } \\
\text { participants }\end{array}$ & 8 questions ouvertes & & $\mathrm{x}$ & \\
\hline & $\begin{array}{l}\text { Perception de la formation } \\
\text { ESF reçue }\end{array}$ & 15 items, Likert à 4 degrés & & $\mathrm{x}$ & \\
\hline Participants & $\begin{array}{l}\text { Sentiment de compétence et } \\
\text { sentiment d'influence face à } \\
\text { des situations problèmes }\end{array}$ & $\begin{array}{l}26 \text { items, Likert à } 4 \text { degrés; } \\
8 \text { items, échelle de } 0 \text { à } 10\end{array}$ & & $\mathrm{x}$ & $\mathrm{x}$ \\
\hline
\end{tabular}

d'efficacité personnelle s'inscrivant dans la théorie sociocognitive [24].

Le tableau 1 propose un récapitulatif des différents questionnaires utilisés auprès des intervenants éducateurs et des participants au cours de l'étude. Une fois remplis et recueillis, les différents questionnaires des participants ont été envoyés par courrier par les coordinateurs de terrain en Lorraine aux coordinateurs de recherche à Paris.

\section{Résultats}

\subsection{La formation méthodologique des intervenants éducateurs en ESF}

\subsubsection{Profil des intervenants éducateurs formés à l'ESF}

22 professionnels ont été formés à l'ESF à Nancy. Ceux-ci sont principalement issus du secteur libéral $(n=7 / 22)$ ou du milieu associatif $(n=5 / 22)$ (Fig. 1). Dix d'entre eux sont médecins (4 praticiens libéraux, 2 praticiens hospitaliers, 2 médecins de la MSA, 1 médecin travaillant dans un réseau de santé et 1 médecin sapeur-pompier professionnel). Dix sont Infirmières Diplômées d'État (IDE) (dont 4 travaillant dans un réseau de santé, 2 en libéral, 1 infirmière hospitalière, 1 infirmière de la Protection Maternelle et Infantile (PMI), 1 infirmière pompier et 1 dont l'exercice professionnel n'est pas connu). Ont participé également à la formation méthodologique 1 pharmacien et 1 assistante sociale.

\subsection{2 Évaluation de la formation à la méthodologie de l'ESF reçue par les intervenants éducateurs}

La satisfaction des intervenants éducateurs vis-à-vis de leur formation de trois jours à l'ESF est élevée, avec une satisfaction moyenne de $94 \pm 0,1 \%$ pour l'organisation générale de la formation et une satisfaction moyenne de $87 \pm 0,1 \%$ vis-à-vis des aspects pédagogiques de la formation. En ce qui concerne les aspects pédagogiques de la formation (Tab. 2), les notions présentées sur l'ESF ont paru claires. Les formateurs ont facilité les échanges entre intervenants éducateurs et ces derniers ont considéré que la diversité professionnelle constituait un enrichissement. La variété des approches éducatives proposées a été également appréciée. Cependant, deux intervenants éducateurs ont regretté un manque de formation sur les techniques pédagogiques, d'animation et de communication.

Les intervenants éducateurs ont souligné d'autres points positifs: la formation a été "enrichissante», "adaptée aux besoins » et elle a «permis de mettre à jour (les) connaissances»d'un d'entre eux sur les «premiers secours/réa ». Sur la forme, la formation était «concrète», «pratico-pratique», «axée sur l'animation», «synthétique» et «bien organisée». Les formateurs ont été qualifiés de "pédagogues».

Mais d'autre part, certaines faiblesses ont été pointées. Un des intervenants éducateurs a considéré que la représentativité des divers secteurs professionnels parmi les personnes formées était déséquilibrée. Un autre participant a estimé que la formation n'était «pas trop approfondie». Quatre d'entre eux ont exprimé un sentiment de crainte vis-à-vis de l'appropriation de la formation, en raison des «outils à finaliser», qu'il «reste à collecter (...) ou à créer (recréer)», il « reste un temps d'adaptation aux outils» mais aussi à la «formation de l'équipe». Il est souligné à deux reprises qu'un travail en binôme serait souhaitable pour une «appropriation des modules et de la méthode d'animation». D'autres réserves concernent le positionnement du professionnel dans cette formation: "pas facile de passer de soignant à éducateur " familial" ", ou encore la problématique de la vulgarisation des connaissances.

\subsection{3 Évaluation du concept d'ESF par les intervenants éducateurs}

À la suite de la mise en œuvre de la formation ESF auprès des familles, les intervenants éducateurs ont été questionnés sur leur ressenti vis-à-vis du concept de l'ESF. Les résultats sont présentés dans le tableau 3. D'une manière générale, selon les intervenants, les lieux pour dispenser une formation ESF auprès des familles ont paru en totale adéquation avec la 


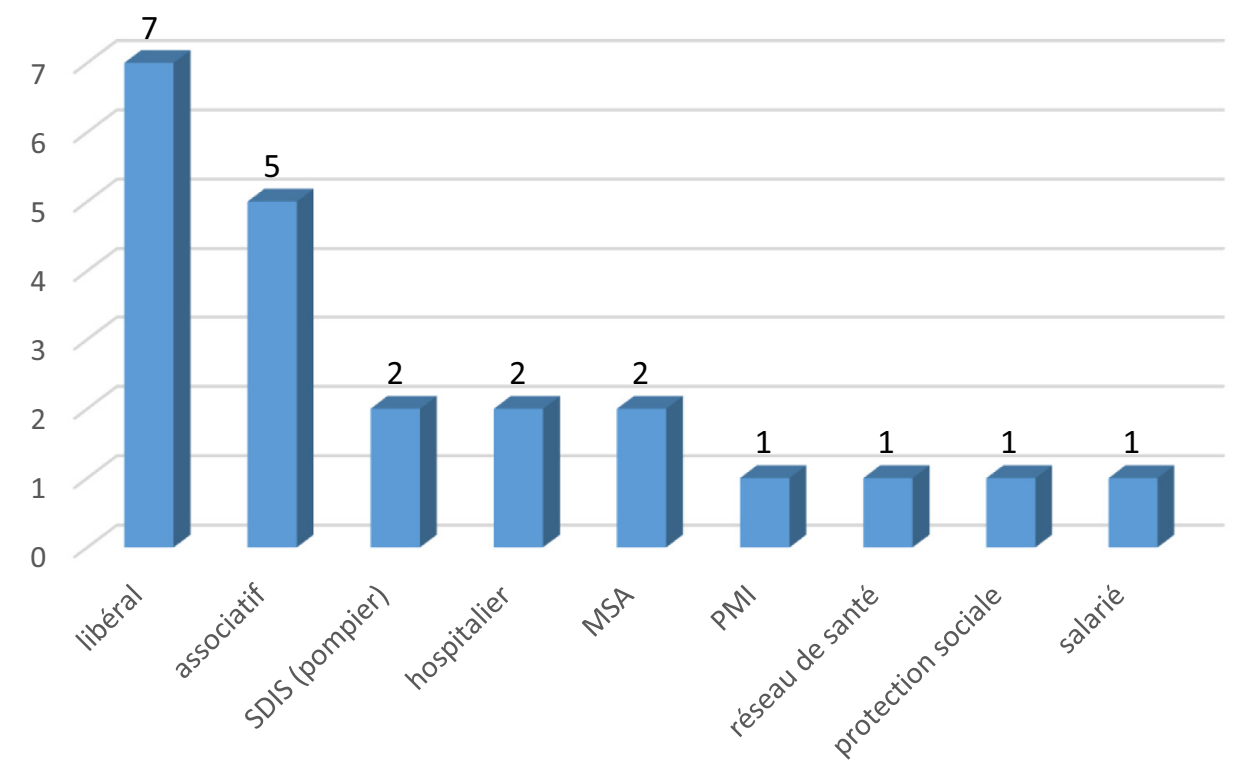

Fig. 1. Secteur d'activité des intervenants ESF $(n=22)$. SDIS : Service Départemental d'Incendie et de Secours ; MSA : membre de la Mutualité Sociale Agricole; PMI : Protection Maternelle et Infantile.

Fig. 1. Activity sector of ESF trainers ( $n=22)$; SDIS: Departmental Fire and Rescue Service; MSA: Agricultural social insurance; PMI: Maternal and Child Protection.

Tableau 2. Satisfaction des intervenants éducateurs vis-à-vis de la formation à la méthodologie de 1'ESF $(n=22)$.

Table 2. Trainers' satisfaction with training in ESF methodology $(n=22)$.

Concernant les aspects pédagogiques

Les notions présentées sur l'ESF m'ont paru claires

Les formateurs ont proposé des approches éducatives variées pour m'entraîner aux situations que je serai amené

L'organisation et la conduite de la formation par les formateurs ont bien reflété les principes pédagogiques qu'ils 20 enseignent (exemplarité)

philosophie du projet; les mises en situations étaient pertinentes au regard des thématiques abordées. De même, les modules du tronc commun et les modules spécifiques au choix ont correspondu aux besoins de l'ensemble des familles. Ils ont nuancé leur propos en estimant que les éléments constitutifs des modules ne permettaient pas d'atteindre toutes les compétences attendues. Trois intervenants éducateurs ont suggéré de prévoir du temps supplémentaire afin de pouvoir aborder toutes les thématiques auprès des participants. Quatre autres intervenants ont estimé que le temps de préparation de la formation ESF était «chronophage». D'autre part, cinq intervenants auraient souhaité des supports écrits à distribuer en fin d'atelier afin que les participants gardent une trace écrite des modules.
Tous les intervenants ont estimé, par ailleurs, que la formation ESF était innovante sur le plan des éducations en santé. Leur intérêt pour cette formation était de :

- connaitre l'ESF en tant que professionnel et être acteur de prévention (se sentir utile) (6 réponses);

- toucher une autre population de patients plus diversifiée que la leur (5 réponses);

- retrouver les notions de base et de bon sens sur la santé (4 réponses);

- travailler en pluridisciplinarité (3 réponses);

- diversifier leurs activités (2 réponses);

- mettre à jour les connaissances des professionnels et celles des participants à l'ESF ( 2 réponses);

- «éviter la consommation médicale excessive» (1 réponse). 
Tableau 3. Évaluation, par les intervenants éducateurs, du concept d'ESF.

Table 3. Evaluation, by trainers, of the ESF concept.

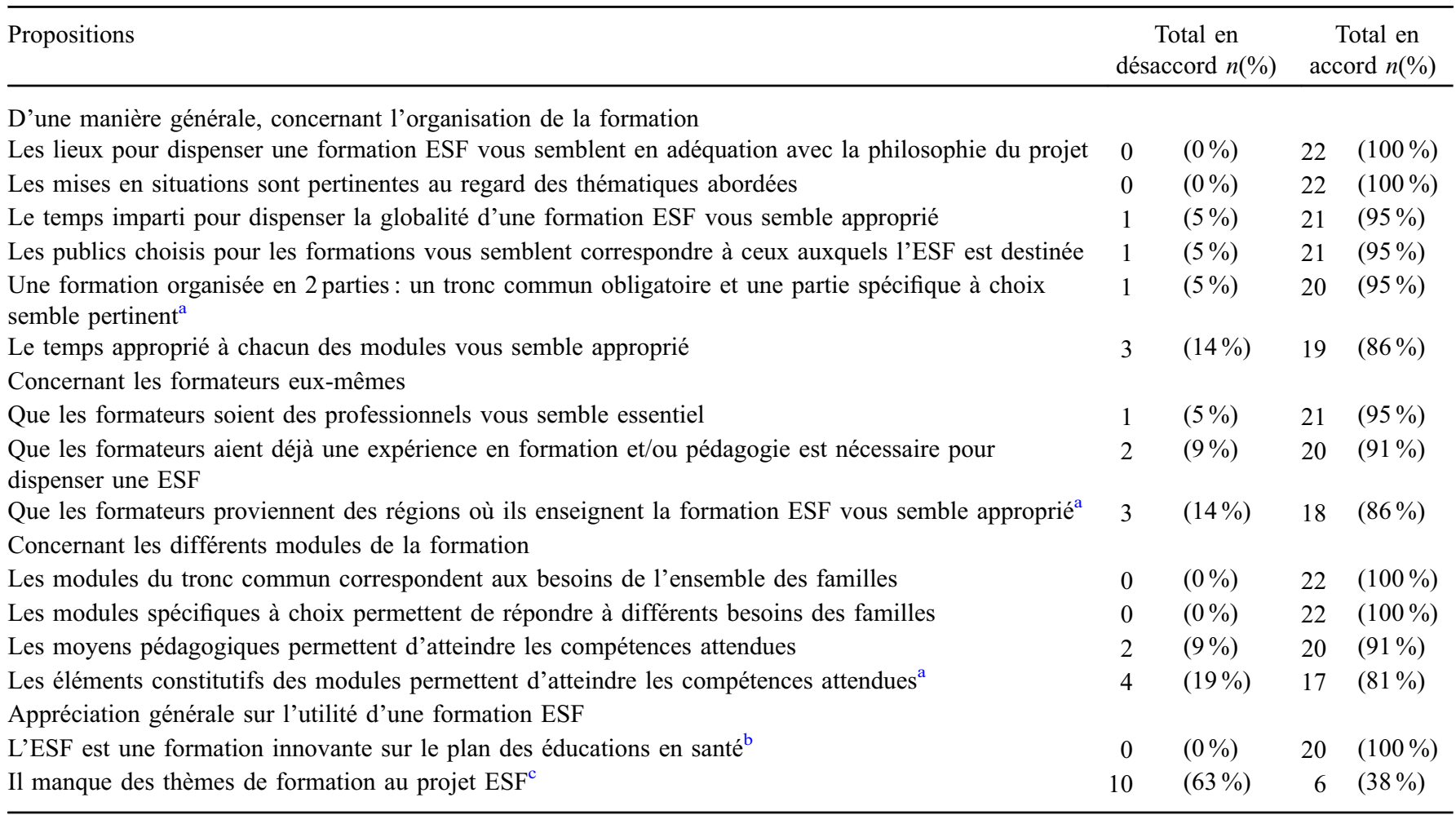

$n=22$.

${ }^{\mathrm{a}} n=21$.

${ }^{\mathrm{b}} n=20$.

${ }^{\mathrm{c}} n=16$.

Selon eux, les thèmes qui pourraient manquer à la formation ESF sont: l'hygiène alimentaire ( 5 réponses), l'hygiène de vie (3 réponses), l'activité physique (2 réponses), la sexualité ( 2 réponses), la pédiatrie ( 2 réponses) et les premiers secours (AVC, infarctus, allergies) (2 réponses). Autres réponses (1 réponse à chaque fois) : les problématiques d'addictions, de douleurs abdominales, de maladies contagieuses et les thématiques du handicap, du travail et de la précarité en lien avec la santé.

\subsection{Résultats de la mise en œuvre de la formation ESF auprès des participants}

\subsection{1 Évaluation de la mise en œuvre de la formation} ESF par les intervenants éducateurs

Parmi les 22 intervenants éducateurs formés à la mise en place d'une formation ESF, 6 sont intervenus à Lunéville, 3 à Montmédy et 9 à Stenay $(n=18)$.

Trois intervenants ont répondu dans chaque ville aux différents questionnaires proposés immédiatement après la formation des participants $(n=9$ au total). Trois intervenants de Lunéville n'ont pas répondu.

En ce qui concerne la mise en place de la formation pour la ville de Lunéville, la promotion de la formation ESF a été réalisée auprès de multiples professionnels du territoire par un travailleur social de la MSA, en partenariat avec une infirmière formée à l'ESF de la Maison des Réseaux de Santé du Pays Lunévillois (MRSL); des plaquettes d'information et des affiches leur ont été également remises. Cependant, le recrutement s'est avéré difficile: les intervenants s'attendant à plus que 5 participants sur les 12 personnes qui avaient initialement reçu un courrier d'invitation.

À Montmédy, le recrutement des participants a été effectué avec l'aide du centre social et de la crèche. À Stenay, les salariés du réseau ADOR et de la MSA ont apporté leur soutien pour mettre en place la formation au sein de l'établissement scolaire. Dans les formations ESF auprès d'adultes (Lunéville et Montmédy), aucune difficulté n'a été signalée en ce qui concerne la mise en place de la formation.

À Stenay, auprès d'un public d'adolescents, les intervenants répondants ont signalé que le module spécifique «agir en tant que jeune » n'était pas assez détaillé et qu'il manquait un certain nombre de documents.

À Lunéville et Montmédy, le kit pédagogique était, selon les intervenants, adapté aux modules de tronc commun ; par contre, le matériel concernant la pédiatrie semblait insuffisant et inadéquat (bébé factice non-adapté pour le mouchage de nez).

L'organisation des modules et le temps qui leur était imparti a semblé adapté, voire un peu trop long, notamment pour les situations d'exception à Stenay. 
Tableau 4. Perception de la formation ESF par les participants ${ }^{\mathrm{a}}$.

Table 4. Perception of the ESF program by participants.

\begin{tabular}{|c|c|c|c|c|}
\hline \multirow{2}{*}{$\begin{array}{l}\text { Propositions } \\
\text { Les formateurs ont écouté avec intérêt ce que j'avais à dire }{ }^{b}\end{array}$} & \multicolumn{2}{|c|}{$\begin{array}{c}\text { Total en désaccord } \\
n(\%)\end{array}$} & \multicolumn{2}{|c|}{$\begin{array}{c}\text { Total en accord } \\
n(\%)\end{array}$} \\
\hline & 0 & $(0 \%)$ & 32 & $(100 \%)$ \\
\hline Le lieu où s'est déroulée la formation a été satisfaisant & 1 & $(3 \%)$ & 32 & $(97 \%)$ \\
\hline Le temps consacré à cette formation est suffisant & 1 & $(3 \%)$ & 32 & $(97 \%)$ \\
\hline Les échanges d'expériences entre les participants ont été constructifs & 1 & $(3 \%)$ & 32 & $(97 \%)$ \\
\hline Les formateurs m’ont donné la possibilité de m’exprimer sur mes expériences & 1 & $(3 \%)$ & 32 & $(97 \%)$ \\
\hline Les supports utilisés par les formateurs étaient adaptés & 2 & $(6 \%)$ & 31 & $(94 \%)$ \\
\hline Cette formation à la santé correspond à mes besoins et à ceux de ma famille & 2 & $(6 \%)$ & 31 & $(94 \%)$ \\
\hline Cette formation me permettra d'améliorer la prise en charge de ma santé et celle de ma famille & 2 & $(6 \%)$ & 31 & $(94 \%)$ \\
\hline Les temps consacrés aux explications et ateliers pratiques ont été suffisants ${ }^{b}$ & 2 & $(6 \%)$ & 30 & $(94 \%)$ \\
\hline Cette formation me permettra de mieux parler de ma santé au quotidien dans ma famille & 3 & $(9 \%)$ & 30 & $(91 \%)$ \\
\hline J'ai appris des choses que je ne connaissais pas & 4 & $(12 \%)$ & 29 & $(88 \%)$ \\
\hline Cette formation m'a donné envie de passer le brevet de secouriste & 5 & $(15 \%)$ & 28 & $(85 \%)$ \\
\hline Globalement, cette formation a répondu à mes attentes & 5 & $(15 \%)$ & 28 & $(85 \%)$ \\
\hline Cette formation pourrait être proposée comme option au bac ${ }^{c}$ & 6 & $(19 \%)$ & 25 & $(81 \%)$ \\
\hline
\end{tabular}

$n=33$.

${ }^{\text {a }}$ Scores exprimés sur 10 .

${ }^{\mathrm{b}} n=32$.

${ }^{\mathrm{c}} n=31$.

L'ambiance pendant les modules a été jugée très bonne dans l'ensemble par les intervenants, sauf auprès des adolescents où elle a été «mouvementée et bruyante».

L'opinion des participants que les intervenants ont pu recueillir a été globalement positive : « acquisition de nouvelles compétences », « remise à niveau appréciée ». Les intervenants jugent qu'il y a eu «beaucoup d'échanges», que le «groupe (était) très participatif et motivé ». Par contre, dans la formation ESF auprès des adolescents, les intervenants ont estimé que «le module 1 n'est pas adapté au public » et que « les ateliers en fin de journée font que le public est moins attentif».

La majorité des intervenants $(n=6 / 9)$ s'est déclarée prête à recommencer une nouvelle formation ESF, ayant jugé l'«expérience intéressante». Ils ont estimé que la formation méthodologique en amont avait été aidante $(n=5 / 9)$ «pour le contenu et (la) modalité du déroulement», avec la réserve que cela «demande du temps de préparation».

Les principales différences par rapport à d'autres formations que les intervenants auraient déjà effectuées sont notamment: «les actes d'urgence» jugés «très pratiques », «le public ciblé (jeunes mamans)» à Lunéville. À Montmédy, la différence réside dans « la finalité globale du programme».

\subsubsection{Profil des participants}

Les participants ont été 33 au total, se répartissant en un groupe de 5 adultes à Lunéville, 12 adultes à Montmédy et un groupe de 16 adolescents à Stenay.

Le groupe de Lunéville ( 5 adultes) était exclusivement féminin. Trois femmes avaient des enfants, soit en bas âge, soit adolescents. Toutes étaient sans emploi, mais quatre d'entre elles avaient un projet professionnel clairement défini : préparation au concours d'auxiliaire de puériculture, agrément d'assistante maternelle et formation d'auxiliaire de vie. Sur ces cinq participantes, une seule vivait sur le territoire de Lunéville, les autres personnes ayant fait le déplacement avec leur véhicule personnel ou en bus depuis Badonviller $(\approx 35 \mathrm{~km})$ et Fontenoy la Joute $(\approx 25 \mathrm{~km})$.

Les 12 participants de Montmédy, dont 11 femmes et 1 homme, étaient des professionnels issus en majorité du secteur de la petite enfance: 4 assistantes maternelle, 4 auxiliaires de petite enfance, 2 enseignants, 1 puéricultrice et 1 ouvrière.

À Stenay, le groupe était composé de 16 adolescent(e)s (15 garçons et 1 fille), tous collégiens en classe de $4^{\mathrm{e}}$ et internes de l'établissement scolaire de formation par alternance: «Maison Famille Rurale de Stenay».

\subsection{3 Évaluation de la formation ESF par les participants}

La satisfaction moyenne des participants des trois villes visà-vis de la formation ESF s'est avérée élevée $(92 \pm 6 \%)$ ( $c f$. Tab. 4, $n=33)$. La satisfaction la plus importante a concerné l'applicabilité directe au retour à domicile des notions enseignées lors des ateliers pratiques et l'écoute active des intervenants ( $100 \%$ de satisfaction pour ces deux propositions).

La proposition qui a obtenu la moindre satisfaction des participants $(81 \%)$ est que la formation ESF soit proposée comme une option au bac.

\subsection{4 Évaluation du sentiment de compétence et d'influence par les participants}

Les participants se sont sentis, dans l'ensemble et en moyenne, compétents immédiatement après la formation (entre 50 et $100 \%$ ) pour toutes les situations abordées ( $c f$. Tab. 5, $n=32$ ), à l'exception de la compétence de compréhension des comportements d'une personne âgée (59\%). Les participants ont exprimé, en grande majorité, le sentiment 
Tableau 5. Sentiment de compétence perçu par les participants immédiatement après la formation $\operatorname{ESF}\left(n=32^{\mathrm{a}}\right)$.

Table 5. Participants' feeling of competence immediately after ESF program $(n=32)$.

\begin{tabular}{ll}
\hline Je me sens capable de... & $n=32$ \\
\hline Soigner une petite coupure & $>90 \%$
\end{tabular}

Soigner une petite coupure

$>90 \%$

Soigner un mal de gorge

Soigner une toux

Réaliser un bandage, un pansement

Soigner une phlyctène

Organiser une pharmacie de premiers soins à la maison

Réagir en cas de malaise d'une personne

Soulager les brûlures d'estomac

Surveiller et faire baisser la température d'un enfant

$>80 \%$

Mettre une personne en PLS

Savoir quoi faire pour mon entourage en cas de canicule, de grand froid

Trouver les informations nécessaires à mes questions sur la santé

Prendre en charge et calmer les pleurs d'un bébé

Savoir agir en cas de chute

Soigner une piqûre d'insecte

Calmer une douleur articulaire

Soigner un rhume, une rhinite allergique

Mettre en place à domicile des mesures pour éviter une chute

Commencer un massage cardiaque

Donner des conseils de santé à ma famille et mon

entourage

Prendre en charge les coliques d'un bébé

Soigner une poussée de bouton de fièvre

Orienter un copain qui a des problèmes d'addiction ou

psychologique

Démarrer les premiers soins pour une foulure

Gérer les médicaments de mes parents (âgés)

Comprendre le comportement d'une personne âgée

${ }^{\text {a } U n ~ p a r t i c i p a n t ~ d e ~ L u n e ́ v i l l e ~ n o n-r e ́ p o n d a n t . ~}$
Tableau 7. Sentiment de compétence perçu par les participants 9 mois après la formation $\operatorname{ESF}\left(n=17^{\mathrm{a}}\right)$.

Table 7. Participants' feeling of competence 9 months after ESF program $(n=17)$.

\begin{tabular}{cc}
\hline Je me sens capable de... & $n=17$ \\
\hline Organiser une pharmacie de premiers soins à la maison & $100 \%$
\end{tabular}

Réaliser un bandage, un pansement

Mettre une personne en PLS

Gérer les médicaments de mes parents (âgés)

Savoir quoi faire pour mon entourage en cas de canicule, de grand froid

Réagir en cas de malaise d'une personne

Soigner une petite coupure

Soigner une piqûre d'insecte

Démarrer les premiers soins pour une foulure

Soigner un mal de gorge

Calmer une douleur articulaire

Soulager les brûlures d'estomac

Soigner une phlyctène

Prendre en charge et calmer les pleurs d'un bébé

Soigner un rhume, une rhinite allergique

Donner des conseils de santé à ma famille et mon

entourage

$>70 \% \quad$ Soigner une poussée de bouton de fièvre

Trouver les informations nécessaires à mes questions sur la santé

Soigner une toux

Savoir agir en cas de chute

Orienter un copain qui a des problèmes d'addiction ou $\quad>90 \%$

psychologique

Prendre en charge les coliques d'un bébé

Comprendre le comportement d'une personne âgée

Mettre en place à domicile des mesures pour éviter une

chute

Surveiller et faire baisser la température d'un enfant $\quad>70 \%$

Commencer un massage cardiaque

Tableau 6. Sentiment d'influence perçu par les participants sur les situations de santé immédiatement après la formation ${ }^{\mathrm{a}}\left(n=30^{\mathrm{b}}\right)$.

Table 6. Participants' feeling of influence on health situations immediately after ESF program $(n=30)$.

\begin{tabular}{lcccc}
\hline Propositions & $\begin{array}{c}\text { Montmédy } \\
(n=12)\end{array}$ & $\begin{array}{c}\text { Stenay } \\
(n=14)\end{array}$ & $\begin{array}{c}\text { Lunéville } \\
(n=4)\end{array}$ & $\begin{array}{c}\text { Totaux } \\
(n=30)\end{array}$ \\
\hline Savoir utiliser les principaux médicaments & 8,4 & 8,1 & 8,0 & 8,2 \\
Gérer les petits maux de la vie quotidienne & 8,6 & 8,0 & 7,5 & 8,0 \\
Repérer une situation urgente & 8,1 & 7,5 & 8,0 & 7,9 \\
Échanger avec d'autres familles sur les problèmes de santé & 8,5 & 6,5 & 8,0 & 7,7 \\
Utiliser le système de santé et ses instances & 8,1 & 6,7 & 7,5 & 7,4 \\
Gérer une situation urgente & 7,4 & 6,7 & 7,0 & 7,0 \\
Être attentif à la santé des parents âgés & 6,8 & 5,8 & 7,7 & 6,8 \\
Gérer les problèmes de santé courants des bébés et des enfants & 8,4 & 4,6 & 7,0 & 6,7 \\
\hline
\end{tabular}

${ }^{a}$ Scores exprimés sur 10 .

${ }^{\mathrm{b}}$ Un participant de Lunéville et 2 de Stenay non-répondants. 
Tableau 8. Sentiment d'influence perçu par les participants sur les situations de santé 9 mois après la formation ${ }^{\mathrm{a}}(n=17)$.

Table 8. Participants' feeling of influence on health situations 9 months after ESF program $(n=17)$.

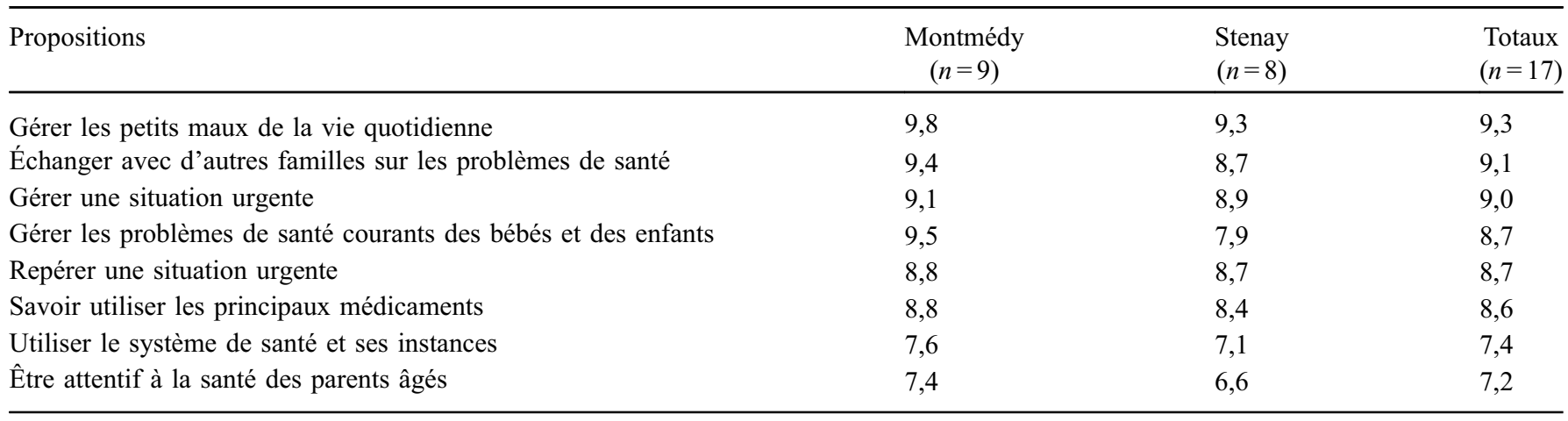

${ }^{\text {a }}$ Scores exprimés sur 10 .

qu'ils pourront influer sur la plupart des situations de santé (scores totaux moyens entre 6 et 10) (cf. Tab. 6, $n=30$ ). Par contre, les adolescents de Stenay ont douté qu'ils aient de l'influence sur la gestion des problèmes courants des bébés et des enfants, et sur le fait d'être attentif à la santé des personnes âgées. Ce dernier résultat correspond aux modules auxquels ils n’ont pas assisté. Cependant, ils ont estimé pouvoir repérer et gérer une situation d'urgence.

À distance de la formation, seuls les questionnaires à 9 mois ont pu être remplis, et 16 participants n'ont pas souhaité répondre ou étaient perdus de vue, dont tous ceux de Lunéville. Les participants se sentent compétents dans les diverses situations de santé (70 à $100 \%$ ) (Tab. 7). Seul le sentiment de compétence perçu à effectuer un massage cardiaque est peu élevé (59\%). Les répondants estiment pouvoir influencer les situations de santé avec des scores tous supérieurs à 6 sur 10 (Tab. 8).

\section{Discussion}

En reprenant les étapes chronologiques de la mise en place de l'ESF, on constate que la formation en amont des intervenants éducateurs a été utile pour qu'ils puissent dispenser les modules ESF. Lors de leur formation méthodologique, les intervenants éducateurs ont beaucoup apprécié l'inter-professionnalité (médecins, IDE, pharmacien, assistante sociale). Il est reconnu que l'inter-professionnalité entraîne une évaluation positive de l'expérience d'apprentissage par des participants à des formations pour adultes [25]. L'inter-professionnalité aurait également un effet bénéfique sur les connaissances, les attitudes, les compétences et les croyances des adultes [25]. Cette modalité de formation apparaît donc primordiale dans le dispositif de l'ESF dont elle constitue un critère de qualité.

Après la mise en œuvre de l'ESF, les intervenants éducateurs ont indiqué qu'ils étaient prêts à réitérer l'expérience; ils ont estimé, dans leur majorité, que l'ESF est une formation innovante dans le champ des éducations en santé. Cependant, ils ont suggéré un soutien plus important sur la pédagogie, l'adaptation des modules aux populations rencontrées, une plus grande organisation dans la préparation de la formation ESF et l'apport de nouvelles thématiques.
Les participants, satisfaits de leur formation ESF, se sont sentis, dans l'ensemble, compétents dans la prise en charge de problèmes de santé quotidiens, avec un sentiment de pouvoir influer sur chaque situation. On peut ainsi émettre l'hypothèse qu'ils ont renforcé ou acquis des savoirs, des savoir-faire et des savoir-être susceptibles de les rendre acteurs de leur santé et de la santé de leur famille au quotidien et dans des situations exceptionnelles aussi bien dans des situations d'urgence (coupure, malaise, bandage et pansement) que pour des problèmes de santé courants (soin de maux de gorge, toux, phlyctène, organisation d'une pharmacie de premiers soins). Ces compétences semblent s'être maintenues dans le temps, voire renforcées. En effet, les 17 participants interrogés 9 mois après l'ESF ont exprimé un sentiment de compétence maximal pour 20 compétences sur 26.

Le double enjeu du transfert de connaissances par les intervenants éducateurs et du maintien de ces connaissances dans le temps est questionné dans le dispositif de l'ESF. On peut estimer que la formation méthodologique n'a pas permis aux intervenants éducateurs de s'approprier suffisamment les techniques pédagogiques et d'animation pour qu'ils aient le sentiment de pouvoir les implémenter aisément. En effet, ils expriment des craintes quant à leur appropriation, et au changement de statut de soignant à éducateur. Une comparaison peut être effectuée avec l'Éducation Thérapeutique du Patient (ETP), dispositif au cours duquel les soignants remplissent un rôle de formateur auprès de patients [26,27]. Ceci nécessite un certain abandon du «pouvoir médical» et une formation spécifique [27]. Les compétences à acquérir ne reposent pas toutes sur des normes ou des recommandations. On peut alors mettre en évidence le côté primordial de l'apprentissage par situation [28], l'utilisation de la stratégie pédagogique de la triade de contextualisation, décontextualisation, recontextualisation (en référence aux travaux de psychologie cognitive) [29], la répétition des actions contextualisées [30,31] et la gestion des émotions [32] qui sont déjà mis en œuvre dans la formation ESF. Dans ce cadre, l'intégration dans la formation ESF des techniques pédagogiques de la simulation serait pertinente [33]. Cette expérimentation ouvre également des perspectives de recherche sur l'intérêt que pourrait avoir l'ESF à entrer dans l'ère du numérique, par l'utilisation, en aval de la formation, d'une 
plate-forme, d'une application, ou encore de jeux intelligents (serious games), par exemple.

Les différents éléments que nous avons recueillis-entre les demandes qui émergent des participants et les remarques des intervenants éducateurs sur les thèmes et contenus de l'ESF nous amènent à nous demander si tous les besoins sont couverts, et comment prendre en compte diverses demandes émanant des participants et des intervenants. En effet, les contenus des modules ESF ont été préalablement formalisés par le biais de données épidémiologiques, d'avis d'experts pluridisciplinaires, de deux enquêtes (l'une nationale et l'autre régionale) et de deux focus groupes [19]. Cependant, à la question «Quels thèmes pourraient manquer, selon vous?», il a été cité la nutrition (alimentation et activité physique), l'hygiène de vie, la sexualité et d'autres maux et situations d'urgences que ceux déjà développés dans les modules actuels (accidents cardiaques, allergies, addictions, douleurs, contagion, handicap). La place et le champ d'action plus ou moins élargis de l'ESF, au regard de ces éléments, sont donc questionnés.

D'autre part, certains éléments de l'ESF manqueraient de pertinence selon les intervenants éducateurs. En effet, certains d'entre eux ont remarqué que le module 1 n'était pas adapté au plus jeune public. Intitulé «Santé et famille au quotidien», ce module vise des compétences comme prévenir, gérer et prendre soin de la santé quotidienne des membres de sa famille. Il est axé sur les maux du quotidien (céphalées, douleurs abdominales et lombaires, vomissements, etc.), sur la pharmacie familiale et la sécurité du domicile. Après évaluation de la première expérimentation de ces ateliers en 2015, et une préconisation d'adaptation du vocabulaire utilisé, il s'est avéré que les adolescents s'investissaient particulièrement dans la gestion de la pharmacie à domicile [19]. En effet, chez les adolescents de notre étude, le sentiment de compétence est relativement élevé quant à l'organisation d'une pharmacie de premiers soins à la maison et c'est également le cas pour la plupart des autres compétences. À l'opposé, les adolescents ne se sentaient pas compétents $(\leq 50 \%)$ principalement pour « donner des conseils de santé à sa famille et son entourage» ou encore "soigner un bouton de fièvre». Il est possible qu'ils n'y voient pas d'intérêt dans l'immédiat. De ce fait, et comme cela est prévu, il convient de promouvoir une adaptation minimale, par les intervenants éducateurs, du contenu des modules en fonction des besoins du public formé et de leurs problématiques spécifiques. Une étude préalable à la formation ESF, afin de connaître les besoins spécifiques du groupe de participants, pourrait être suggérée.

Dans la mesure où cette étude teste l'implémentation de l'ESF dans un territoire, nous pouvons envisager son déploiement. C'est par la dynamique familiale que les notions de bien-être, d'épanouissement, d'hygiène, de propreté, de confort et de sécurité peuvent être amenées [34]. Deux manières d'intégrer ces valeurs sont connues, à savoir par une éducation de base où l'enfant par mimétisme va apprendre cette santé de tous les jours, ou avec des apprentissages informels $[35,36]$. L'ESF offrirait un nouvel angle d'apprentissage auprès de populations-cibles pour lesquelles des dimensions telles que prendre soin de ses proches, transmettre les règles de vie et l'auto-soin ne seraient pas suffisamment ancrées [19]. Il semble donc nécessaire de mettre en place, sur un territoire donné et auprès des familles, un apprentissage afin qu'elles puissent prendre en charge des situations problèmes ou porter secours. Les résultats que nous avons obtenus montrent que l'ESF, développée sur le territoire Lorrain, a constitué - selon les intervenants - un dispositif d'apprentissage adapté, efficace et répondant aux besoins des participants. Ces derniers, à travers leurs réponses aux questionnaires, indiquent que la formation a été efficace et qu'ils ont pu acquérir et maintenir des compétences en santé.

Plus généralement, l'ESF répond aux problématiques actuelles de santé publique, d'éducation en santé, aux attentes du Conseil Économique, Social et Environnemental (CESE) et du Ministère des affaires sociales et de la santé. En effet, l'ESF permet de donner la responsabilité de la prévention aux familles et à la collectivité, et d'agir tôt par une transmission des conseils et des bonnes pratiques au cœur de l'entité que représente la famille. Les individus acquièrent ainsi un pouvoir d'agir et renforcent leurs possibilités de mieux gérer, décider et être acteurs de leur propre santé $[37,38]$. L'ESF pourrait offrir également une réponse innovante à la constatation effectuée par plusieurs rapports des insuffisances, en France, de la promotion de la santé et plus particulièrement de la prévention sanitaire [38-40].

Une réflexion s'ébauche actuellement sur le projet d'une ESF proposée aux parents de jeunes enfants par des personnels de crèches et/ou durant le séjour en maternité pour le module "puériculture » préparant les accouchées et leurs conjoints à la sortie de la maternité, expérimenté sur l'hôpital Nord à Marseille. L'ESF pourrait aussi être positionnée plus en amont dans le parcours de vie. En effet, il pourrait être envisagé de proposer des modules spécifiques d'ESF dans le cadre de la formation qualifiante du baccalauréat: plus de $80 \%$ des participants interrogés dans la présente étude (et $69 \%$ du sousgroupe des adolescents) sont en accord avec cette proposition. Les promoteurs de l'ESF pourraient alors être intégrés en tant que partenaires de la nouvelle politique éducative de santé de l'Éducation Nationale qui met en œuvre depuis la rentrée 2016 un parcours éducatif de santé (PES) pour tous les élèves, de la maternelle au lycée [41].

\subsection{Limites de l'étude}

Cette étude observationnelle d'une phase pilote de formation ESF a été effectuée en Lorraine, ce qui constitue une limite à la généralisation des résultats obtenus qui pourraient être dépendants du territoire où se déroule la formation. D'autre part, les groupes de participants sont hétérogènes. En effet, Lunéville, Montmédy et Stenay se différencient en termes d'accès aux services de santé (notamment, seule la ville de Lunéville dispose d'un centre hospitalier) et de démographie (respectivement 20, 100, 2200 et 2800 habitants), de milieu plus ou moins rural, notamment pour Montmédy et Stenay. De même, les participants à la formation ESF sont diversifiés : les professionnels de la petite enfance à Montmédy ont des connaissances initiales plus importantes en santé que des jeunes en insertion professionnelle (dans des métiers de la petite enfance également) à Lunéville, ou encore des adolescents scolarisés en collège. De plus, les modules spécifiques choisis par les trois groupes de participants ne sont pas identiques: "puériculture et pédiatrie » à Lunéville et Montmédy, et «agir en tant que jeune » à Stenay. Aucun groupe n'a choisi le module «personnes âgées 
et gérontologie ", d'où probablement, le faible sentiment de compétence à comprendre le comportement des personnes âgées. L'hétérogénéité et le nombre restreint de participants sont probablement dus au recrutement, laissé à la charge des structures accueillant la formation ESF, se basant sur leur propre réseau d'usagers. Cela confère cependant à l'implémentation de cette étude un caractère concordant avec les réalités de terrain.

Nous ne disposons pas d'éléments objectifs sur la façon dont se sont déroulés les modules d'ESF. En effet, seules les perceptions des intervenants et des participants nous permettent d'estimer que les modules d'ESF se sont bien déroulés. Aucun évaluateur extérieur n'a effectué d'observation de la formation.

Nous avons choisi d'analyser les données de façon groupée car les participants ont tous été formés par des intervenants éducateurs, eux-mêmes formés à la méthodologie de l'ESF à Nancy pendant les 3 jours de formation. L'intérêt n'était pas d'établir un comparatif entre les groupes, qui sont noncomparables, mais de décrire la mise en œuvre de l'ESF dans trois communes du territoire Lorrain. À ce titre, cette étude pilote a été établie selon l'objectif principal d'estimer la faisabilité du programme et non spécifiquement son effet au moyen d'évaluations longitudinales. Ainsi, les sentiments de compétence et d'influence n'ont pas été questionnés en amont de la formation. Une évaluation de son évolution sera effectuée lors des futures investigations.

Par ailleurs, les questionnaires de sentiment de compétence et d'influence n'ont techniquement pas pu être recueillis auprès des participants à 3 mois. De plus, il n'a pas été possible d'effectuer une analyse longitudinale de l'évolution - immédiatement à la fin du programme à 9 mois - du sentiment de compétence et d'influence sur les situations problèmes chez les participants par défaut d'identification des participants au moment du remplissage des questionnaires.

\section{Conclusion}

La faisabilité de l'expérience de développement de l'ESF sur le territoire Lorrain, grâce au soutien de la MSA, souligne l'intérêt de diffuser ce modèle de formation en santé à plus grande échelle. Suite à cette étude pilote novatrice, des réflexions sur des améliorations pédagogiques et organisationnelles sont en cours. Le défi consistera ensuite à faire connaître cette éducation auprès des institutions, de mobiliser des financements pérennes, de former de futurs intervenants éducateurs volontaires et de proposer une communication de l'existence de l'ESF auprès d'un large public. L'ESF permettrait dans ces conditions de compléter l'offre existante en éducations en santé, constituées de l'éducation pour la santé, de l'éducation thérapeutique du patient et de l'éducation à porter secours. Ce dispositif d'ESF a pour vocation d'être présent sur le territoire afin de permettre à un grand nombre d'individus d'en bénéficier, notamment dans les déserts médicaux et chez les familles distantes des services d'urgence. L'apport de connaissances et de compétences de soins aux familles répondrait à des enjeux sanitaires et sociaux, dont une amélioration de l'éducation en santé traditionnelle et une maîtrise du volume d'activité des soins d'urgence et de premier recours. Sur le plan de la recherche, on pourrait tenter de vérifier à moyen et long terme le nombre de recours aux soins d'urgence et/ou de consultations non planifiées chez les participants ayant suivi une formation ESF. Il serait alors possible de mettre en évidence l'efficacité de cette intervention sur l'accès aux soins d'urgences et de premier recours.

Remerciements. Aux intervenants éducateurs et participants des villes de Lunéville, de Stenay et de Montmédy. À la Mutualité Sociale Agricole pour son soutien.

Conflits d'intérêts. Les auteurs déclarent n'avoir aucun lien d'intérêt concernant les données publiées dans cet article.

\section{Abréviations}

ADOR Réseau territorial Appui, Dispositif et Outils pour les équipes de premiers Recours

AVC Accident vasculaire cérébral

CESE Conseil économique social et environnemental

ESF Éducation à la santé familiale

ETP Éducation Thérapeutique du Patient

IDE Infirmier diplômé d'état

Inpes Institut national de prévention et d'éducation pour la santé

IPCEM Institut de perfectionnement en communication et éducation médicales

LEPS Laboratoire éducations et pratiques de santé

MFR Maison familiale rurale

MRSL Maison des réseaux de santé du pays Lunévillois

MSA Mutuelle sociale agricole

PES Parcours éducatif de santé

PLS Position latérale de sécurité

PMI Protection maternelle infantile

UNAF Union nationale des familles

\section{Références}

1. Evans RG, Barer ML, Marmor TR, eds. Why are some people healthy and others not?: The determinants of the health of populations. Hawthorne (NY): Transaction Publishers; 1994, $401 \mathrm{p}$.

2. Wilkinson R, Marmot M. Les déterminants sociaux de la santé : les faits. $2^{\mathrm{e}}$ éd. OMS bureau Europe; 2004, 40 p.

3. McGinnis JM, Williams-Russo P, Knickman JR. The case for more active policy attention to health promotion. Health Aff (Millwood) 2002; 21(2):78-93.

4. Commission des déterminants sociaux de la santé. Combler le fossé en une génération. Instaurer l'équité en santé en agissant sur les déterminants sociaux de la santé. Rapport final. Genève OMS; 2009, 24 p.

5. Cambon L, Ferron C. Opinion de la Fédération nationale de l'éducation et la promotion de la santé sur le Rapport « Nouvelles approches de la prévention en santé publique». Santé publique 2011; 23(2):143-149.

6. Lasbeur L, Thélot B. Mortalité par accident de la vie courante en France métropolitaine, 2000-2012. Bull Epidemiol Hebd 2017; $1: 2-12$. 
7. Direction de la recherche, des études, de l'évaluation et des statistiques. L'état de santé de la population en France. Rapport 2011. Paris; 2011, 338 p.

8. Fédération internationale des sociétés de la croix rouge et du croissant rouge, centre européen de référence pour l'éducation aux premiers secours. Premiers secours: pour une Europe plus sûre : état des lieux et recommandations; 2009, 20 p.

9. Lecroat A. Projection des populations âgées dépendantes. Ministère des affaires sociales, DRESS, dossier solidarité et santé 43. Paris; 2013, 28 p.

10. Observatoire National de la Démographie des Professions de Santé. Des professions de santé en évolution, le rapport 20102011, tome 3. Paris; 2012, 138 p.

11. Regi JC. Un numerus clausus dans l'admission aux études médicales en France a-t-il jamais eu un sens? J med lib PACA 2013; 44:21-23.

12. Bourgueil U. Démographie et ressources humaines en santé. In: Bourdillon F, Brücker G, Tabuteau D. Traité de santé publique. $3^{\mathrm{e}}$ éd. Paris: Lavoisier Médecine; 2016, 716 p.

13. Zeynep O, Penneau A. Analyse des déterminants territoriaux du recours aux urgences non suivi d'une hospitalisation. Document de travail Irdes 72; 2017, 20 p, disponible sur: www.irdes.fr/ recherche/documents-de-travail/072-analyse-des-determinantsterritoriaux-du-recours-aux-urgences-non-suivi-d-une-hospitali sation.pdf (consulté le 25 septembre 2017).

14. Loi $\mathrm{n}^{\circ} 2016-41$ du 26 janvier 2016 de modernisation de notre système de santé, article 67 , chapitre IV du titre III du livre IV de la première partie du code de la santé publique.

15. Durand AC, Gentile S, Devictor B, Palazzolo S, Vignally P, Gerbeaux P, et al. ED patients: how nonurgent are they? Systematic review of the emergency medicine literature. Am J Emerg Med 2011; 29(3):333-345.

16. Richardson LD, Hwang U. Access to care a review of the emergency medicine literature. Acad Emerg Med 2001; 8 (11):1030-1036.

17. Claudet I, De Montis P, Debuisson C, Maréchal C, Honorat R, Grouteau E. Fréquentation des urgences pédiatriques par les nouveau-nés. Arch Pediatr 2012; 19(9):900-906.

18. d'Ivernois J-F, Gagnayre R, Rodary E, Brun N. Éducation des familles à «Porter Soins et Secours» : un nouveau concept dans le champ de l'éducation en santé. Educ Ther Patient/Ther Patient Educ 2010; 2(1):1-6.

19. Riquet S. Thèse de Santé Publique de l'Université Paris 13 : «Éducation à la santé familiale, modélisation et expérimentation d'un nouveau modèle à porter soins et secours ». Paris; 2015, 339 p.

20. d'Ivernois JF, Gagnayre R. L'éducation au secours de la santé. La lettre de la SETE 2006; 10:1-2.

21. Riquet S, Brun N, Frete F, Ammirati C, Gagnayre R, d'Ivernois $\mathrm{J}$-F. L'éducation à la santé familiale, modélisation et expérimentation de nouvelles interventions éducatives à porter soins et secours. L'éducation à la santé familiale auprès d'adolescents. Educ Ther Patient/Ther Patient Educ 2016; 8(2):20107.

22. Mucchielli R. La dynamique des groupes. $19^{\mathrm{e}}$ éd. Paris: ESF Éditeur; 2008, 238 p.

23. Meirieu P. Le plaisir d'apprendre. Paris; 2014, 157 p.
24. Bandura A. Perceived self-efficacy: Exercise of control through self-belief. In: Dauwalder JP, Perrez M, Hobi V, eds. Annual series of European research in behavior therapy, Amsterdam/Lisse (Netherlands): Swets \& Zeitlinger; 1988, vol 2: pp. 27-59.

25. Cooper H, Carlisle C, Gibbs T, Watkins C. Developing an evidence base for interdisciplinary learning: a systematic review. J Adv Nurs 2001; 35:228-237.

26. d'Ivernois JF, Gagnayre R. Apprendre à éduquer le patient : approche pédagogique. École de Bobigny, 5e éd. Paris: Maloine; 2016, 168 p.

27. Gallois P, Vallée J-P, Le Noc Y. Éducation thérapeutique du patient-Le médecin est-il aussi un «éducateur »?. Médecine 2009; 5(5):218-224.

28. Mazur E. Peer instruction - une méthode éprouvée d'enseignement interactif. Paris: PPUR; 2014, 310 p.

29. Tardif J, Meirieu P. Stratégies pour favoriser le transfert des connaissances. Vie pedagog 1996; 98:4-7.

30. Stordeur J. Comprendre, apprendre, mémoriser : les neurosciences au service de la pédagogie. Paris: De Boeck (Pédagogie et Formation); 2014.

31. Giordan A. Apprendre! Paris: Belin; 2002, 342 p.

32. Nivet P, Alby JM, Crocq L. Les réactions émotionnelles chez les décideurs, les sauveteurs et les soignants. Soins Psychiatr 1989; 106/107:18-22.

33. Granry J, Moll M. État de l'art (national et international) en matière de pratiques de simulation dans le domaine de la santé. Haute autorité de santé; 2012, disponible sur : www.has-sante.fr/ portail/jcms/c_930641/fr/simulation-en-sante (consulté le 25 septembre 2017).

34. Cresson G. Le travail domestique de santé. Paris: L'Harmattan; 1995, $352 \mathrm{p}$.

35. Vial J. Histoire de l'éducation. $4^{\mathrm{e}}$ éd. Que sais-je, Paris: PUF; 2004, $128 \mathrm{p}$.

36. Tessier S. Les éducations en santé. Paris: Maloine; 2012, 216 p.

37. Etienne JC, Corne $\mathrm{C}$. Les enjeux de la prévention en matière de santé. Conseil Économique, Social et Environnemental, Paris; 2012, 54 p.

38. Chaire Inpes. «Promotion de la santé » à l'EHESP et participants au Séminaire des 4 et 5 juin 2012. Plaidoyer pour l'action sur les déterminants sociaux de la santé en France: Les recommandations du 5 juin 2012. Rennes: École des Hautes Études en Santé Publique en collaboration avec l'Institut national de prévention et d'éducation pour la santé, le Secrétariat général des ministères chargés des affaires sociales, la Direction générale de la santé, la Société française de santé publique, l'Union internationale de promotion et d'éducation à la santé, et l'Agence régionale de santé Île-de-France; 2013, 6 p.

39. Direction Général de la Santé. Éléments de réflexion pour une politique nationale de santé. 2011-2025. Paris: DGS; 2011, 53 p.

40. Cour des comptes. La prévention sanitaire. Communication à la commission des affaires sociales de l'Assemblée nationale. Cour des comptes: Paris; 2011, 138 p.

41. Circulaire $\mathrm{n}^{\circ}$ 2011-216 du 2 décembre 2011, disponible sur: www.education.gouv.fr (consulté le 25 septembre 2017).

Citation de l'article : de Montfalcon A, d'Ivernois J-F, Riquet S, Frété F, Deutscher J-L, Gagnayre R, Foucaut A-M. Mise en œuvre de l'Éducation à la Santé Familiale (ESF) en Lorraine. Educ Ther Patient/Ther Patient Educ 2018;10:10203. 